\title{
Effect of surface modification of calcium carbonate nanoparticles on their dispersion in the polypropylene matrix using stearic acid
}

\author{
Mahdi Rahmani, Faramarz Ashenai Ghasemi and Gholamhassan Payganeh \\ Mechanical Engineering Department, ShahidRajaee Teacher Training University, Tehran, Iran
}

Received 8 August 2013, Accepted 28 January 2014

\begin{abstract}
In this study, effects of surface modification of calcium carbonate $\left(\mathrm{CaCO}_{3}\right)$ nanoparticles with a single-layer of stearic acid were investigated on dispersion in polypropylene (PP) matrix. Two kinds of $\mathrm{CaCO}_{3}$ nanoparticles (monolayer-coated and uncoated) were used to investigate this effect. All combinations were mixed in a co-rotating twin screw extruder and then were injection molded. After breaking the injection molded parts in liquid nitrogen, effect of surface modification of nanoparticles on their dispersion in the PP matrix was investigated using a field emission scanning electron microscope (FESEM). The results showed good agreement between the measurements made with the TGA analysis and the existing theories. The surface modification of calcium carbonate nanoparticles using a single layer of stearic acid also had an effective influence on improving distribution and dispersion of nanoparticles in the PP matrix.
\end{abstract}

Key words: Polypropylene / calcium carbonate nanoparticles / stearic acid coating / TGA analysis

\section{Introduction}

Calcium carbonate $\left(\mathrm{CaCO}_{3}\right)$ is one of the most favorite fillers used in composite materials such as polypropylene (PP) composites. Low cost, availability, lightness, surface coating facility, easy manufacturing and high load carrying capability are some of the factors that have caused extensive use of this material as reinforcement [1]. Their composites are used in a wide range of applications such as sewer pipes, garden tools, breathing films etc. [2]. Nanoparticles, due to their high surface to volume ratio, can also increase impact strength, modulus and stiffness of the matrix [3-5]

Incompatibility of hydrophilic and energetic surface of calcium carbonate with low energy surface of hydrophobic polymers is a problem that must be solved before using it as a functional filler [6]. Due to high specific surface and high surface energy, $\mathrm{CaCo}_{3}$ nanoparticles have high tendency to agglomeration. In order to reduce surface energy and their compatibility with the PP matrix which have coated them in an appropriate size, a single-layer of stearic acid is necessary. The single-layer of coating reduces surface energy of particle-particle and particlematrix interactions and leads to achieving better mechanical properties of composites $[2,4,7]$. It has been shown that using a complete monolayer coating, appropriate coating for optimum dispersion of nanoparticles in

\footnotetext{
a Corresponding author:

Faramarz_ashenai_ghasemi@yahoo.com
}

a polymer matrix could be obtained $[2,4,8,9]$. Lin et al. [4] found that if only one layer of coating is present, only one peak appears in DTG curve and if the coating is thicker than a single-layer, a shoulder near the peak or may even a new peak appears in the DTG curve.

Although effect of calcium carbonate nanoparticles and their coating on mechanical properties of PP matrices is important, in this work, owing to greater importance of the phenomenon associated with the surface of nanoparticles in mechanical properties of nanocomposites, effect of surface modification of calcium carbonate nanoparticles on dispersion of particles in PP matrix were studied using FESEM microscope images. TGA analysis was used in order to verify the amount of coating measured by the theory relations.

\section{Experiments}

\subsection{Materials}

In this study, PP Homopolymer Moplen HP550J was supplied by Arak Petrochemical Company Iran. Its melt flow index (MFI) was $3 \mathrm{~g} / 10 \mathrm{~min}\left(230{ }^{\circ} \mathrm{C}, 2.16 \mathrm{~kg}\right)$. Calcium carbonate nanoparticles, with trade name of HAKUENKA CC-R, were provided by Shiraishi-Omya GmbH Company Austria which was supplied by Omya Pars Company in Iran. The average particle size of CC-R was determined to be about $80 \mathrm{~nm}$ that was reported 


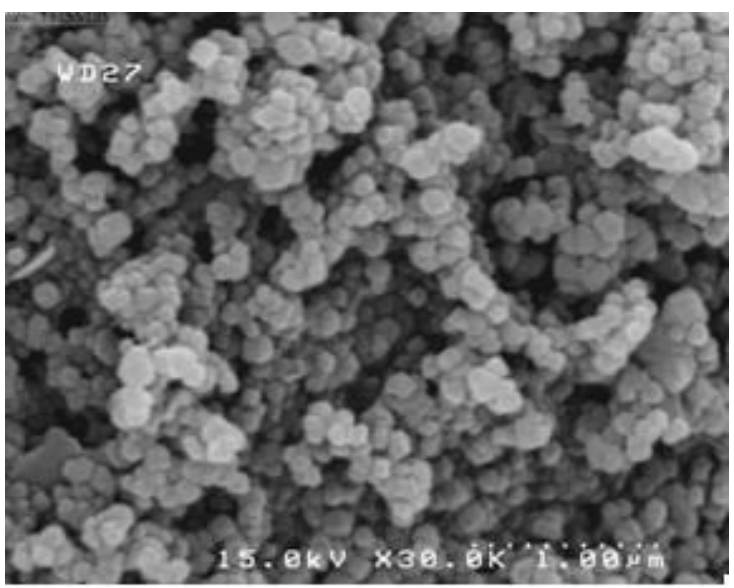

Fig. 1. FESEM micrograph of CC-R.

Table 1. Compositions of the composites ${ }^{\mathrm{a}}$.

\begin{tabular}{lcc}
\hline \multirow{2}{*}{ Abbreviation } & \multicolumn{2}{c}{ Component's Amounts } \\
\cline { 2 - 3 } & $\mathrm{PP}$ & $\mathrm{CaCO}_{3}$ \\
\hline $\mathrm{PP}$ & 100.0 & 0.0 \\
$95 / 5 \mathrm{C}$ & 95.0 & 5.0 \\
$90 / 10 \mathrm{C}$ & 90.0 & 10.0 \\
$90 / 10 \mathrm{CCR}$ & 90.0 & 10.0 \\
85/15C & 85.0 & 15.0 \\
$80 / 20 \mathrm{C}$ & 80.0 & 20.0 \\
\hline
\end{tabular}

"Note: "C" and no letter number denote calcium carbonate and PP in combination, respectively.

by the company. This size was also verified by measuring sizes of the particles by FESEM micrograph, as shown in Figure 1. The stearic acid that was used for coating surface of the calcium carbonate nanoparticles was purchased from Merck Company of Germany with a purity grade of $97 \%$ and product number of 800673 .

\subsection{Sample preparation}

Prior to extruding the compounds, calcium carbonate nanoparticles were dried in an oven for $2 \mathrm{~h}$ at $120{ }^{\circ} \mathrm{C}$. Composite ingredients were mixed together in each case based on weight percentage. Compounding the materials was done using a co-rotating twin screw extruder (ZSK 25 P8.2E WLE). This extruder had six temperature control zones. Unblended pure PP and other blends were prepared using a temperature profile of $160 / 165 / 170 / 175 / 180 / 190{ }^{\circ} \mathrm{C}$. The screw speed was fixed at $250 \mathrm{rpm}$. The $L / D$ ratio of the screws was 40 and $D=25 \mathrm{~mm}$. The extrudate was cooled in a water bath and later granulated into a form ready for injection molding. Injection molding was carried out in temperature profile of $190 / 200 / 210{ }^{\circ} \mathrm{C}$ at injection pressure of 90 bar. Abbreviations of the generated compositions are listed in Table 1. Letter "C" represents weight percent of calcium carbonate nanoparticles and no letter number indicates weight percent of PP. In a compound CCR symbol is used to represent presence of non-coated calcium carbonate nanoparticles.

\subsection{Surface treatment of calcium carbonate nanoparticles}

The received nanoparticles were coated approximately with 2.76 wt\% stearic acid by the manufacturer. To achieve better distribution and dispersion of $\mathrm{CaCO}_{3}$ nanoparticles in PP matrix in order to achieve a singlelayer coating, the received nanoparticles were further coated with stearic acid (between $4-5$ weight percent) to increase their compatibility with the PP matrix and to minimize the interactions among the particles [4]. The coating method was as follows: first, the nanoparticles were mixed in a solution mixture of water and ethanol in the volume ratio of 1:3:1 (nanoparticles: water ethanol). The suspension was stirred by a magnetic stirrer for $1.5 \mathrm{~h}$ to completely wet the particles. Then, the suspension was heated to and maintained at $80{ }^{\circ} \mathrm{C}$. A fixed amount of stearic acid dissolved in the ethanol solvent was gradually added to the suspension drop by drop and stirring continued $1.5 \mathrm{~h}$ for surface reactions. Then the mixture was dried in an oven at $100{ }^{\circ} \mathrm{C}$ for $5 \mathrm{~h}$. This calcium carbonate particles' coating method was also used with small differences previously by Lin et al. [4]. In order to verify the desired coating amount to achieve a single-layer coating of stearic acid on calcium carbonate nanoparticles, $10 \mathrm{~g}$ of calcium carbonate particles was mixed with a $40 \mathrm{ml}$ solution mixture of water and ethanol in volume ratio of $3: 1$; after the coating process using TGA analysis the amount of stearic acid coating of particles was confirmed. For quantitatively determining the amount of stearic acid coating on calcium carbonate nanoparticles, TGA test was done by a TGA device model-1500PL made by Polymer Laboratories England. So after sampling, the samples were heated from room temperature to the temperature of $700{ }^{\circ} \mathrm{C}$ at heating rate of $15^{\circ} \mathrm{C} \cdot \mathrm{min}^{-1}$ under air flow. Weight and rate of weight loss of the sample was measured continuously as a function of temperature.

\subsection{Scanning electron microscopy and phase structure analysis}

Studying of nanocomposites microstructure, dispersion of nanoparticles and their size measurements were done by a Hitachi (S-4160) field emission scanning electron microscopy (FESEM) at $15 \mathrm{kV}$ working voltage. The specimens were obtained by cryogenic fracture. After immersing the sample in liquid nitrogen for more than $20 \mathrm{~min}$, it was broken by hand immediately (less than $3 \mathrm{~s}$ ) after being taken out of liquid nitrogen. To prevent electrical charging during the examination, the SEM samples were sputter-coated with a thin layer of gold. The coating of fracture surface was done by TECHNICS machine, Hummer II model, for $12 \mathrm{~min}$ at $6 \mathrm{kV}$ working voltage and $5 \mathrm{~mA}$ flow under argon gas atmosphere. 


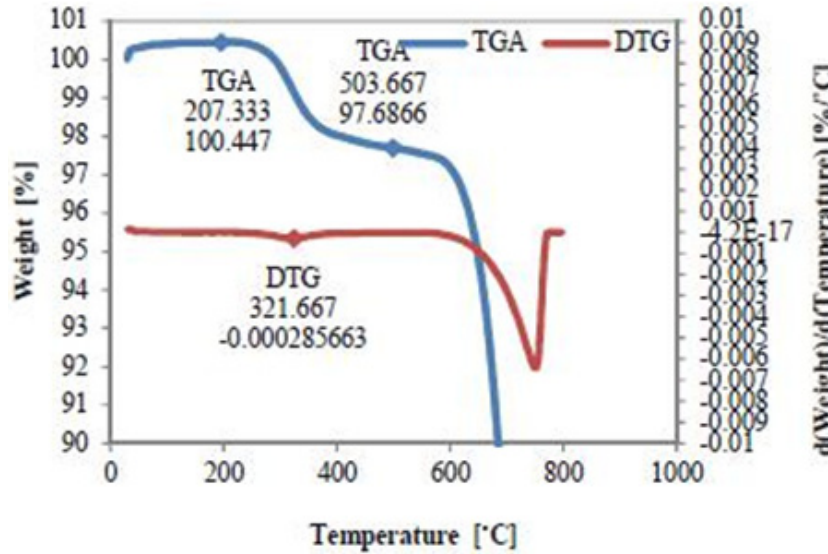

Fig. 2. TGA and DTG curves of initial calcium carbonate nanoparticles.

\section{Results and discussion}

\subsection{Determining stearic acid coverage on calcium carbonate nanoparticles}

Particle-particle interactions lead to a great agglomeration, heterogeneous distribution of fillers, process problems and lower properties of composites. The two main factors that determine particle-particle interaction are size of particle and surface free energy. TGA is a useful technique for detecting organic impurities of calcium carbonate surface and determining optimum coating required for its coverage [6]. The amount of stearic acid needed for a monolayer coating can be also estimated theoretically using the fact that the surface area of nanoparticles was reported by the supplier as $17.0 \mathrm{~m}^{2} \cdot \mathrm{g}^{-1}$ and each $\mathrm{Ca}^{2+}$ ion occupies an area of $0.2 \mathrm{~nm}^{2}[6]$ according to its crystal structure. If each stearic acid's molecule connects one $\mathrm{Ca}^{2+}$ ion and orients vertically to the particle surface [4,6,7], the calculated amount of stearic acid that is needed to produce the complete monolayer coating is about $4.02 \mathrm{wt} \%$. The calculated and TGA results showed good agreement. In order to achieve good dispersion and avoid the negative effect induced by excessive amounts of coating, nanosized calcium carbonate particles were coated with $4-5$ wt\% stearic acid. Figures 2 and 3 show thermal analysis curves of primary calcium carbonate nanoparticles purchased from the factory and single-layer of stearic acid coated calcium carbonate nanoparticles respectively. As can be seen in DTG curves of both analyses, there is only one peak representing a monolayer coating of stearic acid. But given that the most appropriate monolayer coating is a complete monolayer coating equivalent to the maximal weight percent of stearic acid coating on calcium carbonate nanoparticles to achieve a monolayer coating [4], the amount of stearic acid coating on calcium carbonate nanoparticles purchased from the factory was not sufficient according to the amount calculated by the theory equations. Theory relations proposed 4.02 weight percent of coverage while calculating height difference in the peak at approximately $330{ }^{\circ} \mathrm{C}$ temperature in the

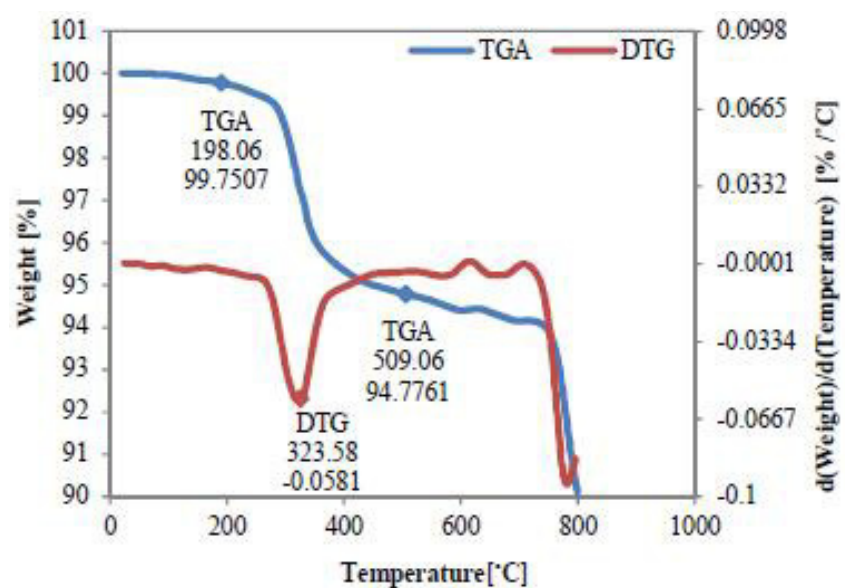

Fig. 3. TGA and DTG curves of the calcium carbonate nanoparticles after coating with complete monolayer of stearic acid.

TGA curve. This was equivalent to elimination of stearic acid from the surface of calcium carbonate particles in the thermal analysis; the initially coated stearic acid was calculated as 2.76 weight percent.

Beginning and end points of peak at TGA curves were specified. In order to achieve complete monolayer coverage of stearic acid on calcium carbonate nanoparticles, approximately 2.5 weight percent of new cover were set upon calcium carbonate nanoparticles until eventually around 5 weight percent of stearic acid coating available on nanoparticles used in this study. In order to verify this amount of coverage, the peak height difference of TGA curve in Figure 3 was calculated that showed approximate number of 4.98 weight percent coating of stearic acid. TGA curve shows the sample weight loss as a function of temperature. Weight loss of up to $500{ }^{\circ} \mathrm{C}$ temperature was low and at temperatures above $500{ }^{\circ} \mathrm{C}$ the intensive weight loss was observed. These results indicated that calcium carbonate nanoparticles could be used as a filler for many thermoplastics because of most thermoplastic processing temperature of below $400{ }^{\circ} \mathrm{C}$ [10].

\subsection{Characteristics of the composites and nanocomposites phase structure}

Figure 4 shows FESEM images taken from the fractured surfaces with liquid nitrogen of PP nanocomposites filled with calcium carbonate nanoparticles in different weight percentages of filler. Figures 4 a to $4 \mathrm{~d}$ shows states filled with $5,10,15$ and 20 weight percent of $\mathrm{CaCO}_{3}$ nanoparticles coated with a monolayer of stearic acid, respectively.

As can be seen in the images, in all weight percentages of the filler, the articles were well distributed because surface treatment with stearic acid significantly reduced filler surface free energy and particle-particle and particlematrix interaction, which led to better dispersion of the 

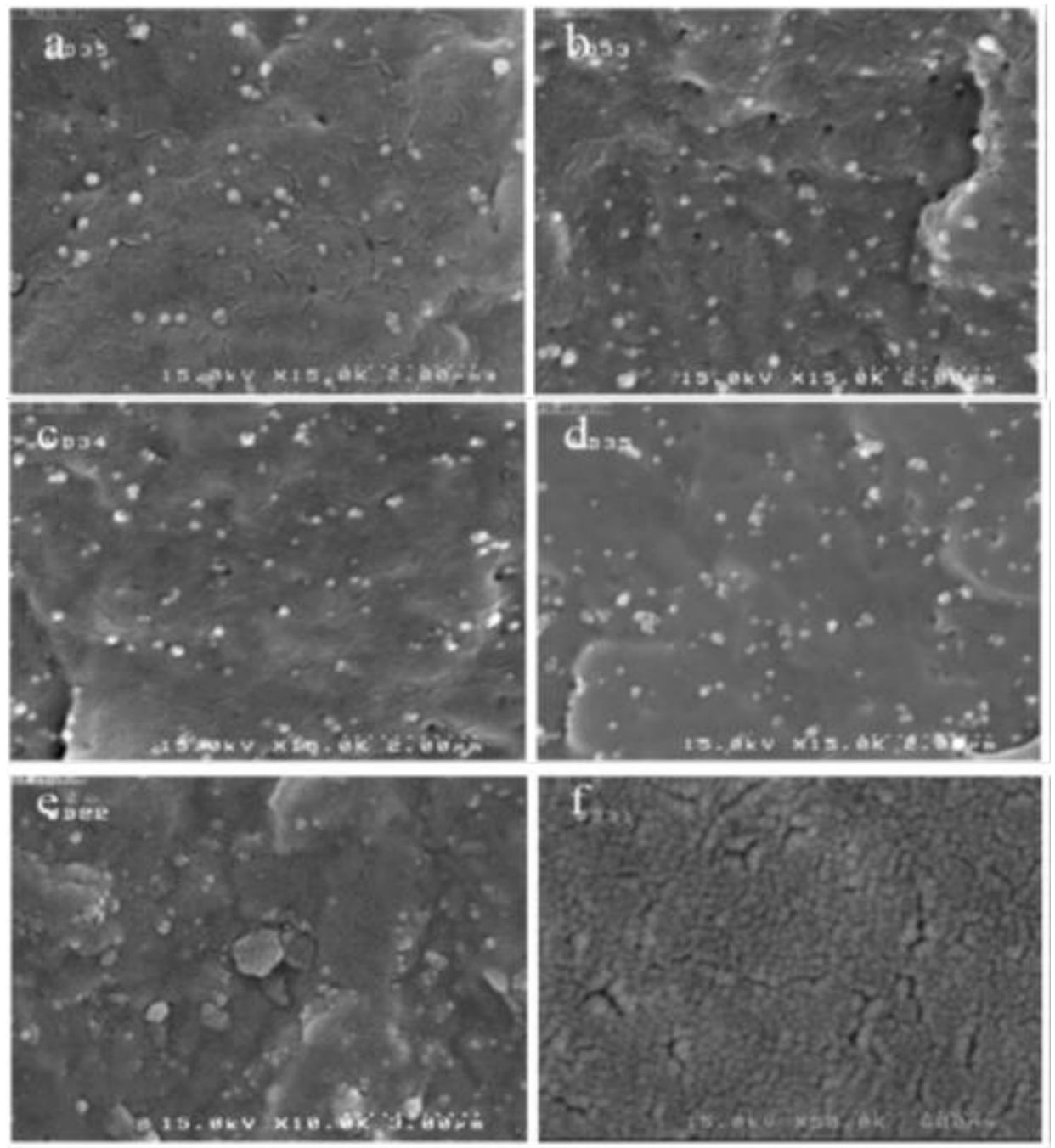

Fig. 4. FESEM images taken from the fractured surfaces with liquid nitrogen of nanocomposites filled with calcium carbonatenanoparticles: (a) $\mathrm{PP} / 5 \mathrm{C}$, (b) $\mathrm{PP} / 10 \mathrm{C}$, (c) $\mathrm{PP} / 15 \mathrm{C}$, (d) $\mathrm{PP} / 20 \mathrm{C}$, (e) $\mathrm{PP} / 10 \mathrm{CCR}$ and (f) PP.

particles in the polymer matrix and reducing the particulates conglomerate $[2,4,7]$. But in states filled with 15 and 20 weight percent of filler (respectively Figs. 4c and 4d) dispersion of small particles was reduced slightly and there was a bit of conglomeration. The reason was that at high density of particles the distance between particles was reduced and there was possibility of interaction between particles' surfaces and hence conglomeration. However, surface coating significantly reduced surface energy; but due to small particles and high specific surface and surface free energy, there was still the probability of particles' sticking together and conglomeration in high percentage of filler $[2,7,8,10]$.

Figure 4e shows composition of PP with 10 weight percent of calcium carbonate nanoparticles without covering. In comparison with Figure $4 \mathrm{~b}$ that had the same weight percent of filler but with monolayer coating of stearic acid it can be seen that distribution and dispersion of the particles were very weak and large conglomeration could be seen in the figure. The reason was high surface energy of the particles and their high tendency to cling to each other. Figure $4 \mathrm{f}$ shows pure PP matrix.

\section{Conclusions}

In this paper, effect of surface modification of $\mathrm{CaCO}_{3}$ nanoparticles on dispersion of particles in PP matrix was studied using FESEM microscope images. TGA analysis was used in order to verify the amount of coating measured by the theory relations. FESEM images taken from the fractured surface with liquid nitrogen of PP nanocomposites filled with $\mathrm{CaCO}_{3}$ nanoparticles showed that coating of the nanoparticles with a monolayer of stearic acid caused better dispersion of particles in the matrix.

Surface treatment of calcium carbonate nanoparticles with stearic acid decreased the particle-particle interactions as well as the polymer-particle adhesion that led to better dispersion of the particles in the polymer matrix and easier separation of particles from the matrix. Furthermore, surface treatment of calcium carbonate 
nanoparticles eliminated incompatibility of hydrophilic and energetic surface of calcium carbonate with a low energy surface of hydrophobic polymers and increased compatibility of these particles with the polymeric matrix.

TGA analysis due to simplicity of sample preparation and testing is a useful technique for detecting organic impurities of calcium carbonate surface and determining the optimum coating required for its coverage.

\section{References}

[1] H. Yang, B. Li, K. Wang, T. EnSun, X. Wang, Q. Zhang, Q. Fu, X. Dong, C.C. Han, Rheology and phase structure of PP/EPDM/SiO2 ternary composites, Eur. Polymer J. 44 (2008) 113-123

[2] A. Kiss, E. Fekete, B. Pukanszky, Aggregation of CaCO3 particles in PP composites: Effect of surface coating, Compos. Sci. Technol. 67 (2007) 1574-1583

[3] ChuanGuo Ma, Yu Liang Mai, Min ZhiRong, Wen Hong Ruan, Ming Qiu Zhang, Phase structure and mechanical properties of ternary polypropylene/elastomer/nanoCaCO3 composites, Compos. Sci. Technol. 67 (2007) 2997-3005
[4] Yong Lin, Haibin Chen, Chi-Ming Chan, Jingshen $\mathrm{Wu}$, High Impact Toughness Polypropylene/CaCO3 Nanocomposites and the Toughening Mechanism, Macromolecules 41 (2008) 9204-9213

[5] Zhang Ling, Wang Zhenghua, Huang Rui, Li Liangbin, Zhang Xinyuan, PP/elastomer/calcium carbonate composites: effect of elastomer and calcium carbonate contents on the deformation and impact behavior, J. Mater. Sci. 37 (2002) 2615-2621

[6] Maged A. Osman, Ulrich W. Suter, Surface Treatment of Calcite with Fatty Acids: Structure and Properties of the Organic Monolayer, Chem. Mater. 14 (2002) 4408-4415

[7] W.C.J. Zuiderduin, C. Westzaan, J. Hue'tink, R.J. Gaymans, Toughening of polypropylene with calcium carbonate particles, Polymer 44 (2003) 261-275

[8] Yong Lin, Haibin Chen, Chi-Ming Chan, Jingshen Wu, The toughening mechanism of polypropylene/calcium carbonate nanocomposites, Polymer 51 (2010) 3277-3284

[9] Yong Lin, Haibin Chen, Chi-Ming Chan, Jingshen Wu, Effects of coating amount and particle concentration on the impact toughness of polypropylene $/ \mathrm{CaCO}_{3}$ nanocomposites, Eur. Polymer J. 47 (2011) 294-304

[10] Chi-Ming Chan, Jingshen Wu, Jian-Xiong Li, Ying-Kit Cheung, Polypropylene/calcium carbonatenanocomposites, Polymer 43 (2002) 298-2992 